\title{
LOCALLY GRADED GROUPS WITH CERTAIN MINIMAL CONDITIONS FOR SUBGROUPS, II
}

\author{
Javier Otal and JUan ManUel PeÑa
}

\begin{abstract}
This paper deals with one of the ways of studying infinite groups many of whose subgroups have a prescribed property, namely the consideration of minimal conditions. If $P$ is a theoretical property of groups and subgroups, we show that a locally graded group $G$ satisfies the minimal condition for subgroups not having $\mathcal{P}$ if and only if either $G$ is a Cernikov group or every subgroup of $G$ satisfies $\mathcal{P}$, for certain values of $\mathcal{P}$ concerning to normalilty, nilpotency and related ideas.
\end{abstract}

\section{Introduction}

This paper deals with one of the most important topics in the study of infinite groups many of whose subgroups have a prescribed property, namely the consideration of minimal conditions in the direction described by the introduction of $[\mathbf{9}]$. Specifically we shall prove:

Theorem. Let $c$ be a nonnegative integer and let $\mathcal{P}$ be one of the following properties: (i) normal; (ii) locally nilpotent; (iii) nilpotent of class at most c; (iv) normal or locally nilpotent; (v) normal or nilpotent of class at most $c_{;}$(vi) $c$-hamiltonion. If $G$ is a locally graded group, then we have:

(1) A minimal non-P subgroup $M$ of $G$ is finite.

(2) $G$ satisfies the minimal condition for subgroups not having $\mathcal{P}$ if and only if either $G$ is a Cernikov group or every subgroup of $G$ satisfies $\mathcal{P}$.

A group $G$ is said to be locally graded if every non-trivial finitely generated subgroup of $G$ has a non-trivial finite image. This weak finiteness condition is made in order to avoid the presence of the Tarski's monsters or the groups described by Ol'sanskii in [5], which show that the result is false without some sort of finiteness condition. It is easy to show that the class of locally graded groups is a local and a residual class of groups and, since abelian and finite groups are clearly locally graded, it follows that such class is very extensive as it contains many different types of groups (see[2, p. 155]). 
The second part of the present Theorem is an extension of some cases of $[9$, Theorem $B]$, where the same conclusion in cases (i)-(v) was proved for $\mathcal{W}$-groups, that is, groups in which every finitely generated non-nilpotent subgroup has a finite non-nilpotent image. This class of groups is subgroup and locally closed, closed under extensions and it is also very ample because it includes locally hyper-(abelian or finite) groups and linear groups. However $\mathcal{W}$-groups form a proper subclass of locally graded groups; indeed the groups constructed in [11] are locally graded but they are not $\mathcal{W}$-groups. In [6] and especially in [7] we have initiated the aforesaid extension from $\mathcal{W}$-groups to locally graded groups adding the $c$-hamiltonian case, which has a different behaviour and will be introduced and explained in the next section. Thus the main result in $[7]$ shows completely the case (i) and the cases (iii), (v) and (vi) when $c=1$. To do this, a fundamental tool is the finiteness of the minimals, which we have included as our first conclusion because it is of interest in its own right. Precedents of that were some of the results obtained in [1] (Corollaries 1 and 2), where the finiteness of a minimal in case (iii) for locally graded groups and in case (ii) for $\mathcal{W}$-groups was proved; the extension of the latter to locally graded groups is immediate since a such minimal is finitely generated and so nilpotent-by-finite. Finally, Ivan [4] has studied the locally nilpotent and the abelian cases under various assumptions, some of which are included in ours.

Throughout our general group-theoretical notation is standard and is taken from [10]. $\mathcal{N}_{c}$ will denote the class of nilpotent groups of class at most $c$; thus $G \in \mathcal{N}_{c}$ if and only if $\gamma_{c+1}(G)=1$. The definitions of minimal non-P group or group satisfying the minimal condition for subgroups not having $\mathcal{P}$ (in short $\operatorname{Min}($ non-P)) can also be interpreted within that context (see [1], [2], [6], [7], [8] or [9]). For our convenience we quote here some well-known facts about nilpotent groups, which will be freely used in what follows: Let $G$ be a nilpotent group. Then $G$ is finite if and only if $G / G$ is finite ([10]; Corollary to 2.26). If moreover $G$ is finitely generated, then $G$ is polycyclic and so it is a residually finite group satisfying Max and contains a characteristic torsion-free subgroup of finite index ([10];Part 1 pp. 65-66, Corollary to 9.31 and Part 2 pp. 138139). If furthermore $G$ is torsion-free, then $G$ is residually a finite $p-g r o u p$ for every prime $p$ ([10, Corollary to 9.38$])$.

\section{2. - - Hamiltonian groups}

In a certain sense, this type of groups was first considered in [1], at least with this degree of generality. Let us go to the definitions. If $c$ is a nonnegative integer, a group $G$ is said to be a $c$-hamiltonian group if every subgroup of $G$ is either normal in $G$ or an $\mathcal{N}_{c}$-group. Thus $O$-hamitonian groups are exactly hamiltonian (or Dedekind) groups and their structure is well-known by results due to Dedekind and Baer (see[1, 2.1.2], for example), and 1-hamiltonian groups are usually known as metahamiltonian groups. Clearly the $c$ - hamiltonian groups form a class which is closed under subgroups and quotients. It is not 
hard to show that this class is also locally closed, which will be fundamental in what follows. For, assume that $H$ is locally a $c$-hamiltonian group that contains a subgroup $K$ which is not normal in $H$ and such that $\gamma_{c+1}(K) \neq 1$. Then $K$ contains a finitely generated non $-\mathcal{N}_{c}$ subgroup, say $L$, and there cxist elements $x$ in $K$ and $h$ in $H$ such that $x^{h} \notin K$. The subgroup $\langle L, h, x\rangle$ of $H$ is $c^{-}$ hamiltonian so that its subgroup $S=\langle L, x\rangle$ must be normal in it, In particular $x^{h} \in S$, which is a contradiction showing our claim. A similar argument gives some information about the form of a minimal $c$-hamiltonian group $G$ : it can be generated by a finitely generated non $-\mathcal{N}_{c}$ subgroup, say $L$, and by elements $x$ and $h$ in $G$ such that $x^{h} \notin\langle L, x\rangle$.

Only a few facts are known about $c$-hamiltonian groups in general. For example a locally graded c-hamiltonian group is a $\mathcal{W}$-group ([1, Proposition 2]). Since there are locally graded 1-hamiltonian groups which are not locally nilpotent $([\mathbf{1}$, Theorem D and VII $])$, we cannot include $c$-hamiltonian groups among the classes considered by Phillips Wilson (see [9, p. 45]) and we shall need to provide different proofs to solve this case. This will be carried out with the aid of later classification results as those of [2] and [8]. To apply these results we have needed to show a property of $c$-hamiltonian groups, which is interesting in its own right. The case $c=1$ was originally showed by Cernikov in $[3, \mathrm{p}, 511]$ and later by Bruno-Phillips in [1, Corollary 3$]$.

(2.1). Let $H$ be a locally graded c-hamiltonian group. Then either $H$ belongs to $\mathcal{N}_{c}$ or $H^{\prime}$ is finite.

Proof: We assume that $H \notin \mathcal{N}_{c}$ and then we have to show that $H^{\prime}$ is finite. Since $H$ is a $\mathcal{W}$-group, $[\mathbf{1}$, Theorem $\mathrm{B}]$ assures that $H^{\prime}$ is finite in the case where $H$ is not locally nilpotent. Thus we assume that $H$ is locally rilpotent.

First at all, we claim that if $H$ is finite, then $\left|H^{\prime}\right|$ is divisible by at most two different primes (one of which is necessarily 2). For, if $N$ is a minimal non $\cdots \mathcal{N}_{c}$ subgroup of $H$, then $N$ is a normal p-subgroup of $H$ and $H / N$ is hamiltonian. Therefore the derived group of $H / N$ has order at most two, that is, $\left|H^{\prime} N / N\right| \leq 2$ and our claim follows.

Assume that $H$ is infinite and let $K$ be a finitely generated non $-\mathcal{N}_{c}$ subgroup of $H$. Then again $\left|H^{\prime} K / K\right| \leq 2$. Furthermore $K$ is nilpotent and so $H^{\prime} \cap K^{\prime}$ and $H^{t}$ are finitely generatcd. Thus we may choose a finitely generated subgroup $V$ of $H$ containing $K$ such that $V^{\prime}=H^{\prime}$. Therefore, replacing $H$ by $V$, we may assume that $H$ is finitely generated and so nilpotent. Since $H / H^{\prime}$ is infinite, $H$ contains a torsion-free normal subgroup $L$ such that $H / L$ is finite. For each integer $m \geq 1, H / L^{m}$ is a finite nilpotent group and, since $L$ is residually finite and $H \notin \mathcal{N}_{c}$ we see that some $H / L^{m} \notin \mathcal{N}_{c}$. Thus, replacing $L$ by this $L^{m}$, we may assume that $H / L \notin \mathcal{N}_{c}$. As we remarked above, $\left|(H / L)^{\prime}\right|$ is divisible by at most two primes. It is not hard to see that, replacing $L$ by some adequate power if necessary, we may choose a prime $q$ such that $\left|\left(H / L^{q^{n}}\right)^{\prime}\right|$ is a $q^{\prime}$-number for every $n \geq 0$. Since $L / L^{q^{n}}$ is always a $q$-group, it follows that $H^{\prime} \cap L=H^{\prime} \cap L^{q^{n}}$ 
for every $n \geq 0$ and then $H^{\prime} \cap L=1$ because $L$ is residually a finite $q$-group. Therefore $H^{\prime}$ is finite.

\section{Proof of the Theorem}

Proof of (1): As we said in the introduction, it remains to show the cases (iv)-(vi). In any case we note that $M$ is finitely generated. For our convenience we fix some additional notation: In what follows if $p$ is a prime, $M_{p}$ will denote a normal subgroup of $M$ of index $p$. The existence of images of $M$ with arbitrary finite orders will be assured in case of $M / M^{\prime}$ being infinite, because, in such a case, $M / M^{\prime}$ will have an infinite cyclic image.

Cases (iv) and (v). Assume that $M$ is infinite. Choose $x$ in $M$ and $g$ in $G$ such that $x^{g} \notin M$. By the case (ii) $M$ contains proper non locally nilpotent subgroups and if $H$ is one of them, then $H$ is normal in $G, M=H\langle x\rangle$ by minimality and so $M^{t} \leq H$. Clearly $M / M^{s}$ must be infinite; otherwise a non locally nilpotent subgroup $K$ of $M$ with $M^{\prime} \leq K \leq M$ and $[M: K]$ maximal would be finite by the case (ii). Thus $M$ has finite images with arbitrary orders. If $p$ and $q$ are different primes, then $M=M_{p} M_{q}$ so that at most one of these $M_{p}$ can be normal in $G$ and therefore the others are locally nilpotent (in fact nilpotent, in case (iv), and $\mathcal{N}_{c}$-groups, in case (v)). Hence $M$ is nilpotent. In case (iv), that is a contradiction. In case (v), let $M / L$ be a finite non- $\mathcal{N}_{c}$ image of $M$; the existence of a such image is ensured because $M$ is residually finite. Pick a prime $q$ not dividing $[M: L]$ and such that $M_{q} \in \mathcal{N}_{c}$. Then $M=L M_{q}$ and so $M / L \in \mathcal{N}_{c}$, a contradiction.

Case (vi). By (2.1) every proper subgroup of $M$ is either an $\mathcal{N}_{c}-$ group or a finite-by-abelian group. Since $M$ is finitely generated,[2, Theorem 2] or $[8,1.4$ and Theorem 1] give that $M$ is a finite-by- $\mathcal{N}_{d}$ group where $d=c$, if $c \geq 1$, and $d=1$, if $c=0$. Thus $\gamma_{d+1}(M)$ is finite and $M / \gamma_{d+1}(M)$ is a finitely generated nilpotent group. It follows that $M$ is polycyclic-by-finite and, in particular, $M$ is a residually finite group satisfying Max.

Assume that $M$ is infinite. Then $M / \gamma_{d+1}(M)$ is infinite and it follows that $M / M^{\prime}$ is infinite. Again $M$ has finite images with arbitrary orders. Since $M \notin \mathcal{N}_{c}$, it has a finite non- $\mathcal{N}_{c}$ image, say $M / K$. If $p$ is a prime not dividing $[M: K]$, then $M=K M_{p}$ and then $M_{p}$ cannot be an $\mathcal{N}_{\mathrm{c}}$-group. By (2.1) $\left(M_{p}\right)^{\prime}$ is finite, $M_{p}$ is central-by-finite $([10,4.32$ and 4.35$])$ and $M / C_{M}\left(M_{p}\right)$ is finite. Given $p$ and $q$ different primes not dividing $[M: K]$, we have that $M=M_{p} M_{q}$ and, since $M / C_{M}\left(M_{p}\right)$ and $M / C_{M}\left(M_{q}\right)$ are finite, we find that $M / \zeta(M)$ is finite. Let $M / L$ be a finite image of $M$ whose order is coprime to $|M: \zeta(M)|$; then $M=L \zeta(M)$. Since $M$ is not $c$-hamiltonian, we may choose a subgroup $U$ of $M$ which is neither normal in $M$ nor an $\mathcal{N}_{c}$-group. By the co-primeness condition, $U \subseteq(U \cap L)(U \cap \zeta(M)) \bmod L \cap \zeta(M)$ and from this it follows that $U=(U \cap L)(U \cap \zeta(M))$. Thus $U \cap L$ is neither normal in $M$ nor an $\mathcal{N}_{c}$-group. This is a contradiction because $L$ is $c$-hamiltonian so that $U \cap L$ must be normal in $L$ and this gives that $U \cap L$ is normal in $M$. 
Thus the proof of (1) is now complete. The proof of (2) needs some auxiliary results which we now state. Some of them are, no doubt, known: we include statements here for the reader's convenience without proof.

The first fact is a general property of locally graded groups, which can be found in [6, Lemma 1] in an equivalent form.

(3.1). A finitely generated infinite locally graded group has an infinite descending chain of normal subgroups of finite index.

As a consequence we have that a finitely generated locally graded group $G$ is finite if and only if $G / F(G)$ is finite where $F(G)$ is the finite residual of $G$, that is, the intersection of all normal subgroups of $G$ of finite index.

The next results is due to Cernikov and a proof of it can be found in $[9$, Lemma 20].

(3.2). If $M$ is a finite subgroup of a group $G$ and $\left(G_{i} / i \in I\right)$ is a descending chain of $M$-invariant subgroups of $G$, then

$$
\cap\left(G_{i} M \mid i \in I\right)=\left(\cap\left(G_{i} \mid i \in I\right)\right) M .
$$

The last auxiliary result allows us to easily reduce some cases from locally graded groups to $\mathcal{W}$-groups.

(3.3). Let $\mathcal{X}$ be a subgroup closed class of groups and let $G$ be a locally graded group satisfying $M$ in (non-LX) that contains a (non-trivial) minimal non-LX subgroup $M$, which is finite. Then $G$ is a locally finite group satisfying $M$ in $n$.

Proof: Assume that $G$ has an infinite descending chain $\left(G_{n} \mid n \in \mathbf{N}\right)$ of normal subgroups. Since $M$ is finite, it is easy to show that the descending chain $\left(G_{n} M \mid n \in \mathrm{N}\right)$ is strict unless at only finitely many steps. Therefore, for some $n, G_{n} M$ is an $L \mathcal{X}$-group and so is $M$, a contradiction which shows that $G$ satisfies Min- $n$.

Now let $H$ be a finitely generated subgroup of $G$. As above we find that $\langle H, M\rangle$ satisfies Min-n so that it must be finite by (3.1). Therefore $H$ is finite and then $G$ is locally finite.

Proof of (2): The cases (ii) and (iii) can be reduced to $W$-groups using the part (1) and (3.3) so that they follow from [9, Theorem B].

Cases (iv) and ( $v$ ). Put $\mathcal{X}=$ locally nilpotent, in case (iv), and $\mathcal{X}=\mathcal{N}_{c}$, in case (v). Assume that $G$ has a minimal non normal non- $L \mathcal{X}$ subgroup $M$, which is finite by (1). We claim that $G$ is a $\mathcal{W}$-group so that our result will follow from $[9$, Theorem B] again. Assume that $G$ is not a $\mathcal{W}$-group and take $H$ to be a finitely generated non- $\mathcal{W}$ subgroup of $G$; we may replace $H$ by $(H, M)$ to assume that $M \leq H$. Evidently, $H$ is infinite: from (3.1) $H$ contains an infinite descending chain $\left(H_{n} \mid n \in \mathrm{N}\right)$ of normal subgroups of finite index. 
Since $H$ is not a $\mathcal{W}$-group and $\mathcal{X}$ is a class of $\mathcal{W}$-groups, no term of the above chain can be an $\mathcal{X}$-group so that we may assume that every $H_{n}$ is normal in $G$. Let $\left(G_{i} \mid i \in I\right)$ be the set of all non-trivial normal subgroups of $G$ and let $N$ be the intersection of all them. The cardinal of the family $\left(G_{i} M \mid i \in I\right)$ is infinite and the intersection of its members is $N M$ by $(3.2)$. Obviously no $G_{i} M$ can be an $L \mathcal{X}$-group so that we may assume that every $G_{i} M$ is normal in $G$ and therefore $N M$ is normal in $G$ and we note that $N M$ is contained in $H$.

Clearly $N$ satisfies Min (non- $L \mathcal{X}$ ). If $N$ is not an $L \mathcal{X}$-group, then $N$ is locally finite by (3.3) and (1). In any case $N$ is a $\mathcal{W}$-group and so is $H M$. Now $H$ is not a $\mathcal{W}$-group so that $H / N M$ cannot be a $\mathcal{W}$-group. Since $H / N M$ satisfies Min (non-normal), [7, Lemma 3] gives that $H / N M$ is periodic.

Let $F$ be the finite residual of $H$. As above, using (3.2) within $H$, we find that $F M$ is normal in $G$. In particular $N \leq F M$ and so $H / F M$ is periodic, $H / F M$ is isomorphic to the quotient of a residually finite group by a finite group and therefore $H / F M$ is residually finite, which can be shown by using (3.2). In particular $H / F M$ is locally graded. Since $H / F M$ also satisfies Min (non-normal), [7, Theorem (ii)] gives that $H / F M$ is either abelian or locally finite and therefore, in any case, $H / F M$ is finite. Hence $H / F$ is finite and so is $H$ by the consequence quoted after (3.1), a contradiction which shows our claim and proves these cases.

Case (vi). By (2.1) $G$ satisfies the minimal condition for non finite-by- $\mathcal{N}_{d}$ subgroups where $d=c$, if $c \geq 1$, and $d=1$, if $c=0$. By [2, Theorem 1] or [8, Theorem 1], $\gamma_{d+1}(G)$ is Cernikov. If $G$ is not $c$-hamiltonian, then $G$ satisfies $\mathrm{Min}-n$ by (3.3) and so every locally nilpotent quotient of $G$ is Cernikov (see $[10$, Corollary 2 to 5.27$]$ ). In particular $G / \gamma_{d+1}(G)$ is Cernikov and so is $G$.

\section{References}

1. B. BRUNO, R.E. PHILLIPS, Groups with restricted non-normal subgroups, Math. Z, 176 (1981), 199-221.

2. B. Bruno, R.E. Phillips, On minimal conditions related to Miller-Moreno type groups, Rend. Sem. Mat. Univ.Padova 69 (1983), 153-168.

3. S.N. CERNIKOV, Infinite nonabelian groups in which all infinite nonabelian subgroups are invariant, Ukrain. Math. J. 23 (1971), 498-517.

4. L. IVAN, On groups satisfying the minimal condition for certain subgroups, Period. Math. Hungat 14 (1983), 117-124.

5. A. YU. OL'SANSKII, Infinite groups with cyclic subgroups, Soviet Math. Dokl 20 (1979), 343-346.

6. J. OTAL, J.M. PEÑA, Some minimal conditions for locally graded groups, Pub. Sem. Mat. G. Galdeano serie II, 71 (1985).

7. J. OTAL, J.M. PEÑa, Grupos localmente graduados con ciertas condiciones minimales sobre subgrupos, Actas VII Congresso GMEL Coimbra I 
(1985), 251-254.

8. J. OTAL, J.M. PEÑa, Groups in which every proper subgroup is Cernikovby-nilpotent or nilpotent-by-Cernikov, Arch. Math, to appear.

9. R.E. PHILlips, J. WILsON, On certain minimal conditions for infinite groups, J. Algebra 51 (1978), 41-68.

10. D.J.S. RoBinsoN, Finiteness conditions and generalized soluble groups, Springer-Verlag (1972), Berlin.

11. J.S. WILSON, On periodic generalized nilpotent groups, Bull. London Math. Soc. 9 (1977), 81-85.

J. Otal: Departamento de Matemáticas

Facultad de Ciencias

Universidad de Zaragoza

50009 Zaragoza, SPAIN.

J.M. Peña: Departamento de Matemática Aplicada

Escuela U de I'II

Universidad de Zaragoza

50009 Zaragoza, SPAIN.

Rebut el 8 d'Octubre de 1987 